\title{
INFECÇ̃̃O GÁSTRICA POR HELICOBACTER PYLORI EM PACIENTES SINTOMÁTICOS DA IIHA DE SÃO LUÍS, MA: CORRELAÇÃO ENDOSCÓPICA, ANATOMOPATOLÓGICA E MICROBIOLÓGICA
}

\author{
José de Macêdo Bezerra, Arquimedes V. Vale, Joaquim C. Lobato Filho, \\ Sérgio F. Martins, Arnaldo Lopes Albarelli, Suzanne de Jesus A. Albarelli \\ Freire, Euripedes Gomes de Oliveira e José Carlos Longo
}

\begin{abstract}
Com critérios previamente definidos de inclusāo e prévio consentimento, 26 pacientes consecutivos (19 a 64 anos), com queixas referentes ao aparelbo digestivo superior, foram submetidos à endoscopia digestiva, com biópsia, constando de 8 fragmentos da região antro-pilórica (4 da parede anterior e 4 da posterior). Dois fragmentos destinados à cultura; dois a teste da urease livre; dois para esfregaço corado, todos colbidos em meio de transporte adequado sob refrigeração; dois fragmentos foram imersos em formalina a $10 \%$ para exame histopatológico. Dos 26 pacientes, 25 (96\%) apresentaram infecção pelo Helicobacter pylori por um ou mais dos métodos empregados. Em $16(61 \%)$, dos 26, foram observadas alterações pela endoscopia (gastrite em 11, úlcera péptica em dois e cicatriz de úlcera em três casos). Dos pacientes com gastrite endoscópica, 10/11 (91\%) apresentaram-se positivos, bem como todos (100\%) os portadores de cicatriz ou úlcera péptica. Foi observada estreita relação entre a presença de $\mathrm{H}$. pylori e gastrite crônica em 24/25 (96\%). Corte bistológico corado pela hematoxilina-eosina foi o teste de maior sensibilidade diagnóstica: 24/25 (96\%), seguido pelo teste da urease 23/25 (92\%), esfregaço corado 19/25 (76\%) e cultura 18/25 (72\%). Conclui-se que a prevalência de infecção gástrica por $\mathrm{H}$. pylori em portadores de sintomas é elevada, correlacionando-se com gastrite crônica e ülcera. Exame bistológico corado pela bematoxilina-eosina e o teste da urease são os mais sensiveis no diagnóstico da infecção. Os estudos devem prosseguir para elucidação de mais questões relacionadas à infecção, incluindo-se grupo controle de sintonáticos, por sexo e idade.

Palauras-chaves: Infecção gâstrica. Helicobacter pylori. Gastrite crônica e úlcera. Ilha de São Luís, MA.
\end{abstract}

A presença de bactérias espiraladas na mucosa gástrica humana tem sido assinalada desde o início do século²58. Já em 1915, isolavam-se bactérias do estômago e duodeno de pacientes ulcerosos ${ }^{20}$. Todavia a importância desses achados foi negligenciada até 1983 quando, na Austrália, Barry Marshall e Robin Warren $^{13}$ isolaram, a partir de biópsias gástricas, a bactéria espiralada hoje denominada Helicobacter pylori que atualmente tem recebido grande atenção dos bacteriologistas e grastroenterologistas ${ }^{11}$.

\footnotetext{
Núcleo de Patologia Tropical e Medicina Social do Departamento de Patologia da Universidade Federal do Maranhão, São Luís, MA.

Endereço para correspondência: Prof. José de Macêdo Bezerra. Dept ${ }^{\circ}$ de Patologia/UFMA. Pça Madre Deus 02, 65025-560 São Luís, MA. Fax: (098) 222-5135.

Recebido para publicação em 05/04/95.
}

Warren $^{25}$ detectou estreita correlação entre a presença de bactérias inicialmente denominadas Campylobacter like e achados histopatológicos compatíveis com gastrite crônica. Posteriormente, em estudo realizado em 1984 por Marshall \& Warren ${ }^{14}$, em 100 pacientes, observou-se a presença da bactéria, hoje denominada $H$. pylori, em 95\% dos pacientes com gastrite crônica, em 100\% dos com úlcera duodenal e em $77 \%$ daqueles com úlcera gástrica.

Atualmente, tem-se observado que até 100\% dos pacientes com gastrite crônica apresentam infecção do antro por $H$. pylori, bem como em quase todos pacientes com úlcera péptica ${ }^{21}$. Existem evidências convincentes ligando $H$. pylori aos adenocarcinomas do antro, corpo e fundo gástrico ${ }^{17}$. A prevalência da infecção por $H$. pylori em pacientes com úlcera duodenal está em torno de 95 e 100\%2923. 
Bezerra JM, Vale AV, Lobato Filbo JC, Martins SF, Albarelli AL, Freire SJAA, Oliveira EG, Longo JC. Infecção gâstrica por Helicobacter pylori em pacientes sintomáticos da Ilba de Sâo Luîs, MA: correlação endoscópica, anatomopatológica e microbiológica. Revista da Sociedade Brasileira de Medicina Tropical 29:245-250, maijun, 1996.

Os trabalhos realizados até 1993 apresentam dados suficientes para concluir que úlcera duodenal é predominantemente infecciosa². Entretanto, a associação entre $H$. pylori e úlcera gástrica é menos freqüente: em torno de 70 a 90\% dos pacientes com esta afecção estão infectados pelo $H$. pylor $^{10}$.

Com relação à gastrite, a comunidade mundial aceita uma relação causal nítida com H. pylori ${ }^{24}$. A bactéria é a causa mais comum de gastrite inespecífica não erosiva ${ }^{19}$.

O diagnóstico da infecção pelo H. pylori pode ser feito por métodos diretos (histológico, cultura) ou indiretos (hidrólise da uréia, imunológico) $)^{15}$.

O teste da urease tem se revelado de extraordinária sensibilidade no diagnóstico da infecção por $H$. pylori, no antro gástrico, com índice de até $91,1 \%$ seguido pelo esfregaço corado com 79,4 e cultura com $50 \% \%^{22}$.

Este trabalho objetiva dimensionar a prevalência da infecção por $H$. pylori em adultos, bem como avaliar, em primeiro momento, os métodos diretos e indiretos de diagnóstico disponíveis em nosso meio, correlacionando os achados microbiológicos com diagnóstico endoscópico e histopatológico.

\section{MATERIAL E MÉTODOS}

Foram estudados prospectivamente 26 pacientes atendidos no Ambulatório do Hospital Universitário "Presidente Dutra", da Universidade Federal do Maranhão, no período de 23.08 .93 a 26.10 .93 , com idade superior a 18 anos, com queixa do trato digestivo superior, isentos de tratamento para úlcera ou gastrite, uso de corticóide, antinflamatórios ou antibióticos nos dois meses antecedentes à admissão e residentes na Ilha de São Luís, MA.

Após consentimento prévio e afastadas as contra-indicaçôes, os pacientes foram encaminhados ao serviço de endoscopia do referido hospital, sendo os mesmos submetidos à anestesia do orofaringe com nebulização de lidocaína a $2 \%$ e injetou-se por via EV a associação de $1 \mathrm{ml}(5 \mathrm{mg})$ de diazepam ${ }^{\circledR}$ e $1 \mathrm{ml}$ (50mg) de meperidina (dolantina).

Procedeu-se o exame endoscópico com aparelho OLYMPUS (BF tipe IT20D), com retirada de oito fragmentos de biópsia da mucosa antral, a $2 \mathrm{~cm}$ do piloro, sendo quatro fragmentos da parede anterior e quatro da posterior. Três fragmentos de cada parede foram imersos em caldo BHI e mantidos sob refrigeração até o momento de execução das rotinas bacteriológicas, o que ocorreu sempre dentro do espaço não superior a duas horas. Um fragmento de cada parede foi imerso em formalina a $10 \%$ para rotina histopatológica.

Dois fragmentos mantidos sob refrigeração foram inoculados em "HP Uréia Teste" (fornecido pelo Laboratório de Pesquisa em Bacteriologia da UFMG/FUNDEP) e acompanhamento por até 24 horas para observação de atividade ureásica.

Dois fragmentos mantidos em refrigeração foram semeados em duas placas de meio Belo Horizonte $^{18}$, por rolamento, incubadas a $37^{\circ} \mathrm{C}$ em atmosfera de microaerofilia (obtida com o emprego de Microaerobacter da Probac do Brasil), observadas no $3^{\circ}$ dia. Em caso de negatividade foi prorrogada por até 7 dias. A caracterização de positividade foi feita pela morforlogia colonial, formas características coradas pela carbolfucsina, positividade à prova da catalase, oxidase e urease.

Dois fragmentos oriundos do caldo BHI sob refrigeração foram empregados para confecção de esfregaço (duas lâminas), corado também pela carbolfucsina e examinado em objetiva de imersão.

O material colhido em formalina a $10 \%$ foi processado e corado pela hematoxilina-eosina para diagnóstico histopatológico do antro e presença de $H$. pylori.

\section{RESULTADOS}

Dos 26 pacientes submetidos à endoscopia, $25(96 \%)$ apresentaram infecção por $H$. pylori detectada por um ou mais métodos. Contudo, em apenas $16(61 \%)$ foram observadas alterações endoscópicas, assim distribuídas: gastrite em 11 , com 10 positivos para a bactéria; úlcera péptica bulbar em $2 \mathrm{e}$ cicatriz de úlcera bulbar em 3 , todos infectados. Os 10 (38\%) pacientes que apresentaram endoscopia normal, tiveram exame bacteriológico positivo para H. pylori (Tabela 1). 
Bezerra JM, Vale AV, Lobato Filho JC, Martins SF, Albarelli AL, Freire SJAA, Oliveira EG, Longo JC. Infecção gástrica por Helicobacter pylori em pacientes sintomáticos da Ilha de São Luís, MA: correlação endoscópica, anatomopatológica e microbiológica. Revista da Sociedade Brasileira de Medicina Tropical 29:245-250, maijun, 1996.

Tabela 1- Correlação entre achados endoscópicos e infecção por Helicobacter pylori na mucosa antral de 26 paciente sintomáticos - Ilba de São Luís (MA).

\begin{tabular}{|c|c|c|c|c|c|c|}
\hline \multirow{3}{*}{ Endoscopia } & \multicolumn{4}{|c|}{ H. pylori } & & \\
\hline & \multicolumn{2}{|c|}{ positivo } & \multicolumn{2}{|c|}{ negativo } & \multicolumn{2}{|c|}{ total } \\
\hline & $\Pi^{9}$ & $\%$ & $n^{0}$ & $\%$ & $\mathrm{n}^{\mathrm{o}}$ & $\%$ \\
\hline$\overline{\text { Gastrite do antro }}$ & 10 & 38 & 1 & 4 & 11 & 42 \\
\hline Úlcera pep. bulbar ativa. & 2 & 8 & - & - & 2 & 8 \\
\hline Cicatriz de úlcera bulbar & 3 & 12 & - & - & 3 & 12 \\
\hline Normal & 10 & 38 & - & - & 10 & 38 \\
\hline Total & 25 & 96 & 1 & 4 & 26 & 100 \\
\hline
\end{tabular}

Com relação à histopatologia, $25(96 \%)$ dos pacientes admitidos apresentaram gastrite crônica do antro e 24 (92\%) foram $H$. pylori positivos. Vale ressaltar que o paciente histologicamente normal apresentou exame bacteriológico positivo (Tabela 2).

Tabela 2 - Correlação entre acbados bistopatológicos e infecção por Helicobacter pylori, na mucosa antral de 26 pacientes sintomáticos - Illa de São Luis $(M A j$.

\begin{tabular}{|c|c|c|c|c|c|c|}
\hline \multirow{3}{*}{ Histopatologia } & \multicolumn{4}{|c|}{ H. pulori } & & \\
\hline & \multicolumn{2}{|c|}{ positivo } & \multicolumn{2}{|c|}{ negativo } & \multicolumn{2}{|c|}{ total } \\
\hline & $\mathrm{n}^{\underline{0}}$ & $\%$ & $\mathrm{n}^{\mathrm{o}}$ & $\%$ & $n^{\mathrm{Q}}$ & $\%$ \\
\hline Gastrite crônica & 24 & 92 & 1 & 4 & 25 & $\overline{96}$ \\
\hline Normal & 1 & 4 & - & - & 1 & 4 \\
\hline Total & 25 & 96 & 1 & 4 & 26 & 100 \\
\hline
\end{tabular}

O comportamento dos métodos diagnósticos empregados teve o seguinte perfil: dos 25 pacientes positivos para H. pylori, 24 (96\%) foram diagnosticados pelo exame histopatológico; $23(92 \%)$ pela hidrólise da uréia (teste da urease, cuja positividade se evidencia pela mudança de cor do meio "HP Uréia Test", de amarelo âmbar para róseo); 19 (76\%) por esfregaço corado e $18(72 \%)$ pela cultura (Tabela 3, Figuras 1, 2 e 3).

\section{DISCUSSÃO}

O diagnóstico de infecção da mucosa gástrica por H. pylori, em pacientes com queixas referentes ao aparelho digestivo superior, tem apresentado taxas variáveis de

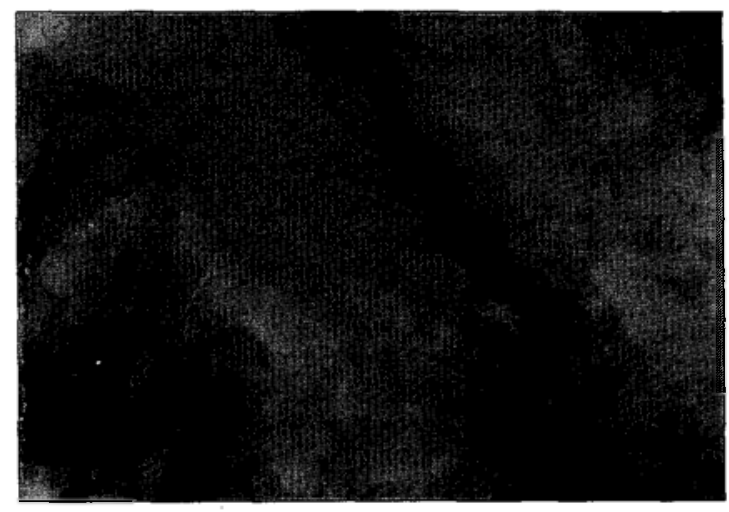

Figura 1 - Aspecto de Helicobacter pylori em corte bistológico da mucosa antral corado pela bematoxilinaeosina (prop. dos autores). Aumento: 100/1,25.

positividade. Solari e cols ${ }^{21}$, em 50 pacientes encontraram $68 \%$ de infectados utilizando o teste da urease pré-formada, bacterioscopia pelo Gram e cultura. Dos pacientes infectados, 91,1\% apresentaram diagnóstico de gastrite crônica em atividade. Em $76 \%$ dos pacientes, foram observadas alterações pelo exame endoscópico. Coelho encontrou positividade de $81 \%$ em pacientes com gastrite endoscópica

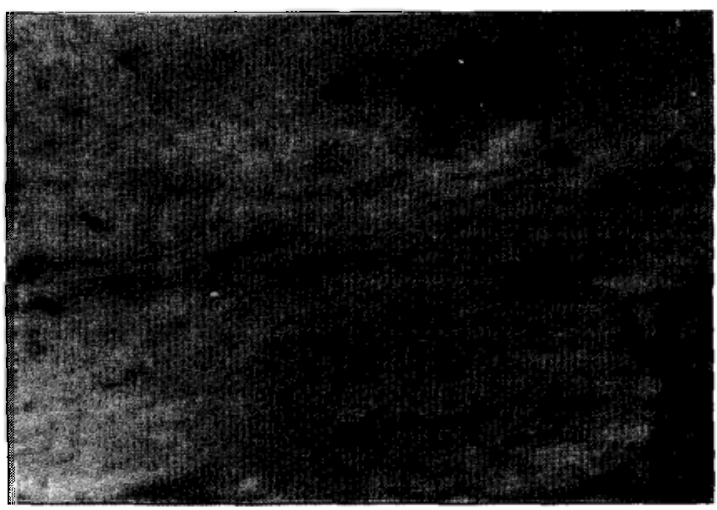

Figura 2 - Aspecto de Helicobacter pylori em muco da mucosa antral atraves de esfregaco curado pela carbofucsina (prop. dos autores). Aumento: 100/1,25.

Tabela 3 - Desenpenbo dos métodos de diagnóstico para Helicobacter pylori em 25 pacientes sintomáticos. infectados, diagnosticados por um ou mais métodos - Ilha de São Luis (MA).

\begin{tabular}{|c|c|c|c|c|c|c|c|c|}
\hline \multirow{3}{*}{ Helicobacter pylort } & \multicolumn{6}{|c|}{ Métodos de diagnóstico } & \multirow{2}{*}{\multicolumn{2}{|c|}{ Cultura }} \\
\hline & \multicolumn{2}{|c|}{ histologia } & \multicolumn{2}{|c|}{ urease } & \multicolumn{2}{|c|}{ esfregaço corado } & & \\
\hline & $\mathrm{n}^{\mathrm{o}}$ & $\%$ & $n^{o}$ & $\%$ & $n^{9}$ & $\%$ & $n^{\circ}$ & $\%$ \\
\hline Positivo & 24 & 96 & 23 & 92 & 19 & 76 & 18 & 72 \\
\hline Negativo & 1 & 4 & 2 & 8 & 6 & 24 & 7 & 28 \\
\hline Total & 25 & 100 & 25 & 100 & 25 & 100 & 25 & $\overline{100}$ \\
\hline
\end{tabular}


Bezerra JM, Vale AV, Lobato Filbo JC, Martins SF, Albarelli AL, Freire SJAA, Oliveira EG, Longo JC. Infecção gâstrica por Helicobacter pylori em pacientes sintomáticos da Ilba de São Luís, MA: correlação endoscópica, anatomopatológica e microbiológica. Revista da Sociedade Brasileira de Medicina Tropical 29:245-250, maijuin, 1996

antral e em $100 \%$ dos portadores de úlcera gástrica e duodenal. A positividade em portadores de gastrite, diagnosticada pelo exame histopatológico, foi de $78 \%$, segundo o mesmo autor.

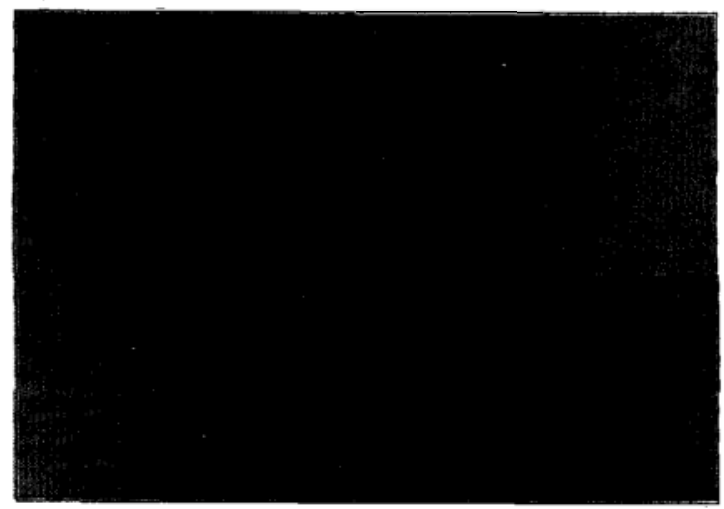

Figura 3 - Aspecto de Helicobacter pylori em esfregaço de cultura pura corado pela carbofucsina (prop. dos autores). Aumento: $100 / 1,25$

Neste estudo, encontra-se taxa de positividade para H. pylori em $96 \%$ dos pacientes submetidos à endoscopia digestiva alta, utilizando a cultura, teste da urease, esfregaço corado pela carbolfucsina e histopatologia; índice, portanto, bem elevado, considerando os espécimes colhidos da mesma área anatômica gástrica, como nos estudos de Solari ${ }^{21}$ e Coelho ${ }^{4}$.

Comparando-se os achados endoscópicos com índices de infecção, verifica-se que 10 dos 11 pacientes com gastrite endoscópica são positivos para H. pylori (91\%), índice também considerado elevado em relação aos outros autores $^{42}$. Os casos de úlcera péptica bulbar, apresentam $100 \%$ de positividade. $O$ indice de positividade nos pacientes com gastrite crônica do antro, em torno de $92 \%$, supera os achados de Coelho ${ }^{4}$. A gastrite inespecífica não erosiva (gastrite crônica) é de diagnóstico histológico e H. pylori tem sido aceito como sua causa mais comum ${ }^{19}$. Dez pacientes apresentaram exame endoscópico normal, porém mostraramse infectados e destes, $9(90 \%)$ apresentaram gastrite crônica ao exame histopatólogico. Em estudo realizado por Coelho ${ }^{4}, 67 \%$ dos pacientes normais à endoscopia foram positivos para $H$. pylori. Solari e cols $s^{21}$ encontraram apenas $25 \%$ de pacientes infectados que se mostraram endoscopicamente normais.
É imperiosa a necessidade de se pesquisar H. pylori por métodos bacteriológicos e/ou histopatológicos ${ }^{21}$. Considerando que o diagnóstico de gastrite crônica é de natureza histopatológica, os pacientes endoscopicamente normais porém com gastrite crônica antral e infecção, reforçam esta necessidade.

Os exames para diagnóstico de $H$. pylori revelam taxas de sensibilidade variável, tais como 93 a 99\% para histologia, 77 a 92 para cultura e 89 a 98 para teste da urease, conforme dados da literatura 121024 . No Brasil, tem se encontrado $91,1 \%$ para urease, $79,4 \%$ para esfregaço corado pelo Gram e prata e 60 a 78 para cultura ${ }^{21}$. Neste estudo, os índices são de $96 \%$ para histologia, $92 \%$ para o teste da urease e $72 \%$ para cultura. O esfregaço corado pela carbolfucsina apresenta $76 \%$ de sensibilidade. Estes índices, portanto, ora são semelhantes à média internacional ou nacional e às vezes a superam. Conclui-se que a prevalência de infecção gástrica por $H$. pylori na Ilha de São Luís, MA é elevada em portadores de gastrite crônica e úlcera bulbar ativa. Estes elevados índices de infecção em portadores de alterações de natureza endoscópica e/ou histopatológica, leva à necessidade de estudos mais amplos, incluindose controles. Os testes da urease, exame direto e histológico, mais sensíveis, sem aparente diferença entre os mesmos. A cultura revela-se um bom método, porém considere-se o custo e as variáveis influentes em seu resultado.

\section{SUMMARY}

With previously defined criteria of inclusion and previous consent, twenty six consecutive patients (19 to 64 years old), with upper digestive symptomes, were submited to endoscopy, with biopsy, constant of eight samples of the antropyloric region (four of the anterior aspect and four of the posterior aspect). Two samples were been for culture; two for free urease test, two for smears; all gathered in adequate transport medium over refrigeration. Two samples immersed in formaline to $10 \%$ for bistopatbologic exam. 25/26 (96\%) of the patients showed infection by $\mathrm{H}$. pylori by means of one or more methods utilized. In 16/26 (61\%), alterations were observed in endoscopy (gastric inflammation in eleven, peptic ulcer in two and ulcer scars in three cases). Of the patients with endoscopic gastric inflammation, presented positive as well as all (100\%) bearers of scar or peptic ulcer. A close relationship was observed between the presence of $\mathrm{H}$. pylori and 
Bezerra JM, Vale AV, Lobato Filho JC, Martins SF, Albarelli AL, Freire SJAA, Oliveira EG, Longo JC. Infecção gástrica por Helicobacter pylori em pacientes sintomáticos da Ilha de São Luís, MA: correlação endoscópica, anatomopatológica e microbiológica. Revista da Sociedade Brasileira de Medicina Tropical 29:245-250, maijun, 1996.

chronic gastric inflammation 24/25 (96\%). Histopatbologic slices stained by bematoxilin-eosin was the test of highest diagnostic sensitivity $24 / 25$ (96\%), followed by urease test 23/25 (92\%), stained smears $19 / 25$ (76\%) and culture 18/25 (72\%). Our conclusion is that the prevalence of gastric infection for $\mathrm{H}$. pylori in symptomatic patients is high, correlated with cbronic gastric inflammation and ulcers. Histopatbologic slices stained by bematoxilin-eosin and free urease test are the most sensitive indicators of the presence of H.pylori. This study should proceed for further elucidation of questions realised and include a control group of symptomatics individuals paired for sex and age.

Key-words: Gastric infection. Helicobacter pylori. Cbronic gastritis and ulcer. São Luis Island, $M A$

\section{REFERÊNCIAS BIBLIOGRÁFICAS}

1. Barthel JS, Everett ED. Diagnosis of Campylobacter pylori infections: "the gold standard" and the standard" and the alternatives. Reviews of Infectious Diseases 12 (suppl): 107-114, 1990.

2. Börsch GMA, Graham DY. Helicobacter pylori. In: Collen MJ, Benjamin SB (eds) Handbook of experimental pharmacology: pharmacology of peptic ulcer disease. Berlin, Springer-Verlag, vol. 99, p. 107-147, 1991

3. Chodos JE, Dworkin BM, Smith F, Van Horn K, Weiss L, Rosenthal WS. Campylobacter pylori and gastroduodenal disease: a prospective endoscopic study and comparison of diagnostic tests. American Journal Gastroenterol 83:1226-1230, 1988.

4. Coelho LGV. Aspectos diagnósticos, patogênicos e terapêuticos da presença de Helicobacter pylori no estômago e duodeno humanos. Tese de doutorado, Universidade Federal de Minas Gerais, Belo Horizonte, MG, 1990.

5. Doenges JL. Spirochetes in the gastric gland of Macacus rhesus and the man without related disease.Archive of Pathology 27:469-477, 1939

6. Freedberg AS, Barron L. E. The presence of spirochetes in human gastric mucous. American Digestive Disease, 7:443-445, 1940.

7. Goodwin CS, Armstrong JA, Chilvers'T, Peters M, Collins MD, Sly L, MacConnel W, Ilaper WES. Transfer of Campylobacter pylori and Campylobacter mustelae to Helicobacter pylori gen. nov. and Helicobacter mustelae comb. nov., respectively. Institute Journal Systematicle Bacterial. 39:397-399, 1989.
8. Goodwin CS, Path FRCP, Worsley BWW. Microbiologia do Helicobacter. In: Dooley $\mathrm{CP}$, Cohen H (eds) Clínicas de Gastrenterologia da América do Norte, Interlivros Edições Ltda, Rio de Janeiro, RJ, vol 1, p.5-19,1993.

9. Graham DY. Campylobacter pylori and peptic ulcer disease. Gastroenterology 96:615-626, 1989.

10. Isenberg J, MacQuaid KR, Laine L. Diseases of the stomach and duodenum: Helicobacter pylori, peptic ulcer disease and gastritis. Int: Yamada $\mathrm{T}$ (ed) Textbook of Gastroenterology, Philadelphia, JB Lippincott, p. 1241, 1991.

11. Kreinitz W. Ueber das Auftreten von Spirochaeten Verschiedener Form in Mageninhalt bei Carcinoma Ventriculi. Deutsch Med Wochenshr 32:872, 1906.

12. Langenberg M1, Tytgat GNJ, Schipper MEI., Rietra PJGM, Zanen HC. Campylobacter-like organism in the stomach of patients and healthy individuals. Lancet 1:1348, 1984

13. Marshall B, Warren JR. Unidentified curved bacilli on gastric epithelium in active chronic gastritis. Lancet 1:1273-1275, 1983.

14. Marshall BJ, Warren JR. Unidentified curved bacilli in the stomach of patients with gastritis and peptic ulceration. Lancet 2:1311-1315, 1984.

15. Montgomery E, Martin DF, Peura DA. Rapid diagnosis of Campylobacter pylori by Gram's stain. American Journal Clinic Pathology 90:606609, 1988.

16. Morris A, Ali MR, Brown P. Campylobacter pylori infection in biopsy specimens of gastric antrum: Laboratory diagnosis and estimation of sampling error. Journal Clinical Pathology 42:727-732, 1989.

17. Parsonnet J. Helicobacter pylory e Câncer Gástrico. In: Dooley CP, Cohen H (eds) Clínicas de Gastrenterologia da América do Norte, Interlivros Edições Ltda. Rio de Janeiro, RJ, vol 1, p. 87-104, 1993.

18. Queiroz DMM, Mendes EN, Rocha GA. Indicator Medium for Isolation of Campylobacter pylori. Journal of Clinical Microbiology 25:2378-2379 1987.

19. Robert ME, Weinstein WM. Patologia Gástrica Associada ao Helicobacter pylori. In: Doley CP, Cohen $\mathrm{H}$ (eds) Clínicas de gastrenterologia da América do Norte, Interlivros Edições Ltda. Rio de Janeiro, RJ, vol 1, p. 57-72, 1993.

20. Rosenow EC, Sanford AH. The bacteriology of ulcer of the stomach and duodenum in man. Journal Infectious Disease 17: 219-226, 1915. 
Bezerra JM, Vale AV, Lobato Filbo JC, Martins SF, Albarelli AL, Freire SJAA, Oliveira EG, Longo JC. Infecção gástrica por Helicobacter pylori em pacientes sintomáticos da Ilha de São Luîs, MA: correlação endoscópica, anatomopatológica e microbiológica. Revista da Sociedade Brasileira de Medicina Tropical 29:245-250, maijun, 1996.

21. Solari CA, Reis, EMF Hofer E, Basílio CA, Válido, CMSR, Luna LI. Pesquisa de Helicobacter (Campylobacter pylori) em pacientes com queixas referentes ao trato gastrointestinal superior. Revista de Microbiologia de São Paulo 21:109$113,1990$.

22. Thillainayagam AV,Arvind AS, Cook RS. Diagnostic efficiency of an ultrarapid room test for Helicobacter pylori. Gut 32:467-469, 1991.

23. Tytgat GNJ, Axon ATR, Dixon MF. Helicobacter pylori, Causal agent in peptic ulcer disease? In: Working Party Report of the World Cogresses of
Gastroenterology, Sydney, Australia, p. 36-45, 1990.

24. Tytgat GNJ, Noach LA, Raws EAJ. Infecção pelo Helicobacter pylori e doença ulcerosa duodenal. In: Doley CP, Cohen H (eds) Clínicas de Gastrenterologia da América do Norte, Interlivros Edições Ltda. Rio de Janeiro, vol 1, p. 125-136, 1993.

25. Warren JR. Unidentified curved bacilli on gastric ephithelium in active chronic gastritis. Lancet $1: 1273,1983$. 